\title{
Microlocal Geometry and Valued Fields
}

\author{
To Professor Sato as a token of admiration \\ by
}

François LOESER

\begin{abstract}
This is an overview of some recent connections between non-archimedean geometry and microlocal analysis, with some emphasis on the motivic Milnor fiber and the properties of tangent cones.

2010 Mathematics Subject Classification: 03C65, 03C98, 11G25, 14B05, 14G20, $14 \mathrm{G} 27$. Keywords: non-archimedean geometry, valued fields, Milnor fiber, tangent cones, microlocal geometry.
\end{abstract}

\section{Introduction}

Microlocal theory of sheaves as developed by Kashiwara and Schapira in their treatise [21] assigns a microsupport to any sheaf on a real manifold. Roughly speaking this is a conic involutive subset of the cotangent bundle which consists of covectors along which the cohomology of the sheaf "does not propagate" in that direction. The prototype of this approach is the theory of the microsupport for hyperfunctions introduced by M. Sato.

In recent years, in particular with the development of motivic integration, the need for developing microlocal geometry over valued fields became more and more apparent. Though we are convinced that a full development of such a theory might take place within the framework of Berkovich spaces (this is one of the motivations behind our work in progress 20. with Hrushovski), and that the final picture should include a satisfactory treatment of $\ell$-adic and motivic sheaves in non-archimedean geometry and have connections with other approaches such as [1] and [33, we shall

This is a contribution to the special issue "The golden jubilee of algebraic analysis".

Communicated by M. Kashiwara. Received January 26, 2010.

F. Loeser: École Normale Supérieure, Département de mathématiques et applications, 45 rue d'Ulm, 75230 Paris Cedex 05, France (UMR 8553 du CNRS);

e-mail: francois.loeser@ens.fr

(C) 2011 Research Institute for Mathematical Sciences, Kyoto University. All rights reserved. 
devote this paper to some quite concrete and down-to-earth results and issues related to microlocal geometry over the $p$-adics and Laurent series fields.

More precisely, we shall focus on two specific topics which have presently reached quite different levels of development, the motivic Milnor fiber in Section 1 and the theory of tangent cones and wave fronts in Section 2. In both cases, we start with the $p$-adic version which provides a useful model before setting the motivic picture. The work presented here is for the most part the result of collaboration with-in chronological order-J. Denef, G. Guibert, M. Merle, R. Cluckers, and G. Comte.

\section{$\S 1$. The motivic Milnor fiber}

\section{$\S 1.1$. The $p$-adic story}

Let $K$ be a finite extension of $\mathbb{Q}_{p}, R$ the valuation ring of $K, P$ the maximal ideal of $R$, and $\bar{K}=R / P$ the residue field with cardinality $q$. Let $\varpi$ be a uniformizing parameter. For $z \in K$ we denote by $\operatorname{ord}(z)$ its valuation, by $|z|$ its absolute value $q^{-\operatorname{ord}(z)}$, and by $\overline{\operatorname{ac}} z$ its angular component, that is, the image of $\varpi^{-\operatorname{ord}(z)} z$ in $\bar{K}$.

Let $f(x) \in K\left[x_{1}, \ldots, x_{n}\right]$ be a non-constant polynomial over $K, \chi: \bar{K}^{\times} \rightarrow \mathbb{C}^{\times}$ a multiplicative character, and $\Phi: K^{n} \rightarrow \mathbb{C}$ a Schwartz-Bruhat function, that is, a locally constant function with compact support. To these data one associates Igusa's local zeta function, which is the meromorphic continuation to $\mathbb{C}$ of the integral

$$
Z_{\Phi}(s, \chi)=\int_{K^{n}} \Phi(x) \chi(\overline{\mathrm{ac}} f(x))|f(x)|^{s}|d x|
$$

for $\Re s>0$, where $|d x|$ denotes the Haar measure on $K^{n}$. Igusa showed that this is a rational function of $q^{-s}$. When $\Phi$ is the characteristic function of $P^{n}$ one sets $Z_{0}(s, \chi)=Z_{\Phi}(s, \chi)$. From now on we suppose that $\chi$ is induced by a character of $\bar{K} \times$.

Our starting point is the following remarkable result proved by Denef in [9] which provides a link between the étale realization of the Milnor fiber and $p$-adic Igusa zeta functions.

1.1. Theorem (Denef [9]). Assume that $f^{-1}\{0\}$ has a resolution with good and tame reduction modulo $P$ and that $f(0)=0$. Then

$$
\lim _{s \rightarrow-\infty} Z_{0}(s, \chi)=(1-q) q^{-n} \sum_{i}(-1)^{i} \operatorname{Tr}\left(\sigma, H^{i}\left(F_{0}, \mathbb{Q}_{\ell}^{\mathrm{a}}\right)^{\chi}\right) .
$$

Here $F_{0}$ is the étale Milnor fiber at 0 of the reduction modulo $P$ of $f, H^{i}\left(F_{0}, \mathbb{Q}_{\ell}^{a}\right)^{\chi}$ is the $\chi$-unipotent part of the action of the local geometric monodromy group on the $\ell$-adic cohomology of $F_{0}$, and $\sigma$ is a suitable lifting of the geometric Frobenius. 
We shall now move on towards the motivic version of the story.

\section{$\S 1.2$. The motivic zeta function}

Let us start by recalling a version of the definition of motivic zeta functions [10, [13. Let $k$ be a field of characteristic 0 and let $X$ be a smooth variety over $k$ of pure dimension $d$. Given an integer $n$, the arc space $\mathcal{L}_{n}(X)$ parametrizes morphisms Spec $k[t] / t^{n+1} \rightarrow X$. Thus, for $K$ a field extension of $k, \mathcal{L}_{n}(X)(K)$ may be canonically identified with $X\left(K[t] / t^{n+1}\right)$. The full arc space $\mathcal{L}(X)$ will only occur occasionally in this text. It may be defined as the inverse limit of the spaces $\mathcal{L}_{n}(X)$.

Consider a morphism $f: X \rightarrow \mathbb{A}_{k}^{1}$ and set, for $n \geq 1$,

$$
\mathcal{X}_{n}(f):=\left\{\varphi \in \mathcal{L}_{n}(X) \mid \operatorname{ord}_{t} f(\varphi)=n\right\},
$$

where $\operatorname{ord}_{t}$ denotes the $t$-adic valuation. Note that $\mathcal{L}_{n}(X)$ is endowed with the canonical $\mathbb{G}_{\mathrm{m}}$-action

$$
a \cdot \varphi(t):=\varphi(a t)
$$

under which $\mathcal{X}_{n}(f)$ is invariant. Furthermore $f$ induces a morphism

$$
f_{n}: \mathcal{X}_{n}(f) \rightarrow \mathbb{G}_{\mathrm{m}}
$$

assigning to a point $\varphi$ in $\mathcal{X}_{n}(f)$ the coefficient $\overline{\mathrm{ac}}(f(\varphi))$ of $t^{n}$ in $f(\varphi)$. Since

$$
f_{n}(a \cdot \varphi)=a^{n} f_{n}(\varphi),
$$

the fiber

$$
\mathcal{X}_{n}^{1}(f):=f_{n}^{-1}(1)
$$

is canonically endowed with a $\mu_{n}$-action, where $\mu_{n}$ is the group scheme of $n$-th roots of unity, $\mu_{n}:=\operatorname{Spec} k[T] /\left(T^{n}-1\right)$.

We fix a $k$-variety $S$ that we endow with the trivial $\mu_{n}$-action and we denote by $K_{0}^{\mu_{n}}\left(\operatorname{Var}_{S}\right)$ the quotient of the free abelian group on isomorphism classes of equivariant $\mu_{n}$-morphisms $Y \rightarrow S$ with $Y$ a variety with good (i.e. such that every orbit is contained in an affine open subset) $\mu_{n}$-action by the additivity relation

$$
[Y \rightarrow S]=\left[Y^{\prime} \rightarrow S\right]+\left[Y \backslash Y^{\prime} \rightarrow S\right]
$$

for $Y^{\prime}$ closed in $Y$ and the additional relation

$$
[A \rightarrow Y \rightarrow S]=\left[A^{\prime} \rightarrow Y \rightarrow S\right]
$$

if $A$ and $A^{\prime}$ are two affine bundles of the same rank over $Y$ with affine $\mu_{n}$-action lifting the same $\mu_{n}$-action on $Y$. This abelian group is naturally endowed with a ring structure induced by the fiber product. 
We then set

$$
\mathcal{M}_{S}^{\mu_{n}}:=K_{0}^{\mu_{n}}\left(\operatorname{Var}_{S}\right)\left[\mathbb{L}^{-1}\right]
$$

with $\mathbb{L}$ the class of the trivial rank one affine bundle over $S$ and

$$
\mathcal{M}_{S}^{\widehat{\mu}}:=\lim _{\mathcal{M}_{S}^{\mu_{n}}}
$$

under the projective system $\mu_{n m} \rightarrow \mu_{n}$.

We can now consider the following series in $\mathcal{M}_{X_{0}(f)}^{\widehat{\mu}}[[T]]$ :

$$
Z_{f}(T):=\sum_{n \geq 1}\left[\mathcal{X}_{n}^{1}(f)\right] \mathbb{L}^{-n d} T^{n}
$$

which is a motivic analogue of Igusa's local zeta function considered in 1.1. Indeed, one can view the $\widehat{\mu}$-action as a natural analogue in the present setting of the multiplicative characters $\chi$ occuring in 1.1 , and when $k$ is a number field, $Z_{f}(T)$ specializes to $p$-adic Igusa functions for large $p$ 's.

Note that $Z_{f}=0$ when $f$ is identically 0 on $X$, so we shall assume the reduced zero locus $X_{0}(f)$ of $f$ is nowhere dense in $X$. We shall now express $Z_{f}(T)$ in terms of a log-resolution $h: Y \rightarrow X$ of $X_{0}(f)$. Recall that this means that $h: Y \rightarrow X$ is a proper morphism with $Y$ smooth such that $h$ induces an isomorphism $Y \backslash E \rightarrow$ $X \backslash X_{0}(f)$, with $E=h^{-1}\left(X_{0}(f)\right)$ a divisor with simple normal crossings. We denote by $E_{i}, i \in A$, the set of irreducible components of $E$. Hence, by definition the $E_{i}$ 's are smooth and intersect transversally.

For $I \subset A$, we set $E_{I}=\bigcap_{i \in I} E_{i}$ and $E_{I}^{\circ}=E_{I} \backslash \bigcup_{j \notin I} E_{j}$. We denote by $\nu_{E_{i}}$ (resp. $\nu_{E_{I}}$ ) the normal bundle to $E_{i}\left(\operatorname{resp} . E_{I}\right)$ in $Y$, by $U_{E_{i}}$ the complement of the zero section in $\nu_{E_{i}}$ and by $U_{I}$ the fiber product of the restrictions of the spaces $U_{E_{i}}, i$ in $I$, to $E_{I}^{\circ}$. There is a canonical $\mathbb{G}_{\mathrm{m}}$-action on each $U_{E_{i}}$ and we consider the diagonal action on $U_{I}$.

We fix $I$ such that there exists $i$ in $I$ such that the multiplicity $N_{i}(f)$ of $f$ along $E_{i}$ is $>0$. Note that the function $f \circ h$ induces a function

$$
\bigotimes_{i \in I} \nu_{E_{i}}^{\otimes N_{i}(f)} \mid E_{I} \rightarrow \mathbb{A}_{k}^{1}
$$

vanishing only over the zero section. We define $f_{I}: \nu_{E_{I}} \rightarrow \mathbb{A}_{k}^{1}$ as the composition of this last function with the natural morphism $\nu_{E_{I}} \rightarrow \bigotimes_{i \in I} \nu_{E_{i}}^{\otimes N_{i}(f)}{ }_{\mid E_{I}}$, sending $\left(y_{i}\right)$ to $\otimes y_{i}^{\otimes N_{i}(f)}$. We still denote by $f_{I}$ the induced morphism from $U_{I}$ to $\mathbb{G}_{\mathrm{m}}$.

Since $f_{I}(\lambda \cdot x)=\lambda^{n} f_{I}(x)$ with $n=\sum_{i \in I} N_{i}(f)$, it follows that $U_{I}^{1}:=f_{I}^{-1}(1)$ is endowed with a $\mu_{n}$-action, so we can consider its class $\left[U_{I}^{1}\right]$ in $\mathcal{M}_{X_{0}(f)}^{\widehat{\mu}}$. 
By using the finite level version of the change of variable for motivic integration (Lemma 3.4 of [1]), one gets

$$
Z_{f}(T)=\sum_{\emptyset \neq I \subset A}\left[U_{I}^{1}\right] \prod_{i \in I} \frac{1}{T^{-N_{i}(f)} \mathbb{L}^{\nu_{i}}-1}
$$

in $\mathcal{M}_{X_{0}(f)}^{\widehat{\mu}}[[T]]$. In particular, the function $Z_{f}(T)$ is rational.

Little is known about the poles of $Z_{f}(T)$, in particular the following conjecture, known as the Monodromy Conjecture, remains largely open, apart from some very specific examples and low dimensional cases, though new significant cases have been proved recently (see [17] and [26]).

1.2. Conjecture. Let $r$ be a rational number. If $\mathbb{L}^{r}$ is a pole of $Z_{f}(T)$, then $\exp (2 i \pi r)$ is an eigenvalue of the monodromy on the stalk of the nearby cycles at some point of $X_{0}(f)$.

\section{$\S 1.3$. The motivic Milnor fiber}

Since we have

$$
\lim _{T \rightarrow \infty} \frac{1}{T^{-N_{i}(f)} \mathbb{L}^{\nu_{i}}-1}=-1,
$$

one deduces immediately from 1.1 that the limit

$$
\mathcal{S}_{f}:=-\lim _{T \rightarrow \infty} Z_{f}(T)
$$

is well-defined in $\mathcal{M}_{X_{0}(f)}^{\widehat{\mu}}$ and that given a log-resolution $h$ we have

$$
\mathcal{S}_{f}=-\sum_{\emptyset \neq I \subset A}(-1)^{|I|}\left[U_{I}^{1}\right] .
$$

If $x$ is a closed $k$-point of $X_{0}(f)$, by restricting to arcs with origin at $x$, one defines similarly $\mathcal{S}_{f, x}$ in $\mathcal{M}_{k(x)}^{\widehat{\mu}}$. By analogy with Theorem 1.1, the virtual object $\mathcal{S}_{f}$ should be the motivic incarnation in $\mathcal{M}_{X_{0}(f)}^{\widehat{\mu}}$ of the complex of nearby cycles $R \psi_{f} \mathbb{Q}_{X}$. Indeed, as proved in 10, its Betti and Hodge realizations are compatible with the semisimplifications of those of $R \psi_{f} \mathbb{Q}_{X}$, the $\widehat{\mu}$-action corresponding to the monodromy action. Motivic vanishing cycles are defined by setting

$$
\mathcal{S}_{f, x}^{\phi}:=(-1)^{d-1}\left(\mathcal{S}_{f, x}-1\right) .
$$

\section{§1.4. Convolution. Applications}

It appears that a certain convolution operator is quite useful for several results regarding the motivic Milnor fiber. If $G$ is a finite group scheme and $X$ and $Y$ are two varieties with good $G$-action, we shall denote by $X \times^{G} Y$ the quotient of the 
product $X \times Y$ by the equivalence relation $(g x, y) \equiv(x, g y)$. The $G$-action on one factor induces a good $G$-action on $X \times{ }^{G} Y$.

For $n \geq 1$, we consider the Fermat varieties

$$
F_{1}^{n}:=\left\{(x, y) \in \mathbb{G}_{\mathrm{m}}^{2} \mid x^{n}+y^{n}=1\right\}, \quad F_{0}^{n}:=\left\{(x, y) \in \mathbb{G}_{\mathrm{m}}^{2} \mid x^{n}+y^{n}=0\right\},
$$

both endowed with their canonical $\mu_{n} \times \mu_{n}$-action. Let $X$ be a variety with a good $\mu_{n} \times \mu_{n}$-action. We set

$$
\Psi_{\Sigma}(X):=-\left[F_{1} \times^{\mu_{n} \times \mu_{n}} X\right]+\left[F_{0} \times^{\mu_{n} \times \mu_{n}} X\right]
$$

in $\mathcal{M}_{\mathbb{C}}^{\mu_{n}}$, the $\mu_{n}$-action being the diagonal one. The construction goes through the projective limit, so we get a group morphism

$$
\Psi_{\Sigma}: \mathcal{M}_{\mathbb{C}}^{\widehat{\mu} \times \widehat{\mu}} \rightarrow \mathcal{M}_{\mathbb{C}}^{\widehat{\mu}}
$$

In fact, given any quasihomogeneous polynomial $Q$, one can generalize the above construction and define convolution operators $\Psi_{Q}$ such that $\Psi_{\Sigma}$ corresponds to the polynomial $\Sigma=x+y$ (see [15]).

The convolution product

$$
*: \mathcal{M}_{\mathbb{C}}^{\widehat{\mu}} \times \mathcal{M}_{\mathbb{C}}^{\widehat{\mu}} \rightarrow \mathcal{M}_{\mathbb{C}}^{\widehat{\mu}}
$$

is then defined by

$$
[X] *[Y]:=\psi_{\Sigma}([X \times Y]) .
$$

It is commutative and associative. It was first constructed at the level of Chow motives in [12], and then on $\mathcal{M}_{\mathbb{C}}^{\widehat{\mu}}$ and its relative versions by Looijenga in [28].

Let $X_{1}$ and $X_{2}$ be smooth varieties of pure dimension $d_{1}$ and $d_{2}$ and consider functions $f_{1}: X_{1} \rightarrow \mathbb{A}^{1}$ and $f_{2}: X_{2} \rightarrow \mathbb{A}^{1}$. Denote by $f_{1} \oplus f_{2}$ the function on $X_{1} \times X_{2}$ sending $\left(x_{1}, x_{2}\right)$ to $f_{1}\left(x_{1}\right)+f_{2}\left(x_{2}\right)$.

When $f_{1}$ and $f_{2}$ have isolated singular points $x_{1}$ and $x_{2}$, Thom and Sebastiani proved that the Milnor fiber with monodromy action of $f_{1} \oplus f_{2}$ at $\left(x_{1}, x_{2}\right)$ is the join of those of $f_{1}$ and $f_{2}$ at $x_{1}$ and $x_{2}$, respectively. The corresponding statement for the Hodge spectrum has been proved by Steenbrink, Varchenko and Saito. It states that the Hodge spectrum of $f_{1} \oplus f_{2}$ at $\left(x_{1}, x_{2}\right)$ is equal to the product of that of $f_{1}$ at $x_{1}$ with that of $f_{2}$ at $x_{2}$.

Using convolution one can prove the following motivic version of the ThomSebastiani Theorem [12, 28], 13], 14, [15]:

1.3. Theorem (Denef-Loeser, Looijenga).

$$
\mathcal{S}_{f_{1} \oplus f_{2},\left(x_{1}, x_{2}\right)}^{\phi}=\mathcal{S}_{f_{1}, x_{1}}^{\phi} * \mathcal{S}_{f_{2}, x_{2}}^{\phi} .
$$




\section{$\S 1.5$. Motivic Milnor fibers of compositions}

The motivic Thom-Sebastiani Theorem 1.3 can be considered as a special case of the following problem: given a two-variable polynomial $P(x, y)$ and two functions $f$ and $g$, compute the motivic Milnor fiber of $P(f, g)$ in terms of those of $f$ and $g$ and data depending on $P$.

In [30, Némethi studied the topological Milnor fiber and monodromy of $P(f, g)$, when the mapping $(f, g)$ is assumed to have a reasonable discriminant. Later, Némethi and Steenbrink [31] were able to compute, under mild assumptions, the Hodge spectrum of composed functions of the form $P(f, g)$. Their result involves the discriminant of the morphism $(f, g)$.

1.4. Question. State and prove a motivic analogue of the results by Némethi and Steenbrink 31] for the motivic Milnor fiber of composed functions of the form $P(f, g)$.

Answering this question in general seems quite difficult. One of the issues one has to face is the following: it seems hard to prove in a general setting that, given a morphism $F: X \rightarrow \mathbb{A}_{k}^{2}$ with $X$ a smooth variety, the induced morphism between arc spaces $\mathcal{L}(X) \rightarrow \mathcal{L}\left(\mathbb{A}_{k}^{2}\right)$ has good properties outside the discriminant locus of $F$. Even the case when the discriminant of $F$ is contained in the coordinate axes seems to require substantial new ideas and some kind of substitutes for microlocal methods. Indeed, over the complex or real numbers, studying the singularities of integrals in the fibers of the morphism $F$ and relating them to the discriminant of $F$ requires tools from $\mathcal{D}$-modules or microlocal geometry (see [2, [27, 32]).

A related problem, somewhat dual, which is also very much open, is the following:

1.5. Question. Find a good motivic analogue of Lê Dung Tráng's result [24], expressing the Milnor fiber of a function $f$ at a singular point $x$ in terms of that of its restriction to a generic hyperplane $\ell^{-1}(0)$ passing through $x$ and combinatorics related to the discriminant of the morphism $(f, \ell)$.

The only general results presently available regarding Question 1.4 assume the functions $f$ and $g$ have no variable in common. In this case, one assigns to $P$ a tree $\tau(P)$ which is obtained by considering the Puiseux expansions of the roots of $P$ (cf. [16]). To certain vertices $v$ of the tree, called rupture vertices, one assigns a quasihomogeneous polynomial $Q_{v}$. The main result of [16] is a computation of the motivic Milnor fiber of $h=P(f, g)$ when $f$ and $g$ have no variable in common, thus extending Theorem 1.3. More precisely, Theorem 4.2 of [16] expresses the $\mathcal{S}_{h}$ as a sum over all rupture vertices of terms of the form $\Psi_{Q_{v}}\left(A_{v}\right)$, with $\Psi_{Q_{v}}$ the 
convolution operator associated to $Q_{v}$, and $A_{v}$ defined inductively in terms of the tree and data depending only on $(f, g)$.

\section{§1.6. Extending $\mathcal{S}_{f}$ to the Grothendieck ring and a motivic analogue of Steenbrink's conjecture}

This subsection is devoted to the exposition of the only significant case where one is able to compute the motivic Milnor fiber of $P(f, g)$ when the functions $f$ and $g$ do have variables in common, namely the case of $h=f+g^{N}$ with $N$ large.

To state the result one first needs to explain the construction of an extension of $\mathcal{S}_{f}$ to a morphism

$$
\mathcal{S}_{f}: \mathcal{M}_{X_{0}(f)} \rightarrow \mathcal{M}_{X_{0}(f)}^{\widehat{\mu}}
$$

that has been constructed by Bittner in [4] using weak factorization and in [14] using motivic integration. This should be viewed as the analogue of considering nearby cycles for complexes of constructible sheaves instead of just the constant sheaf.

Let us start by explaining the construction of $\mathcal{S}_{f}([U])$ when $U$ is a dense open subset of $X$. Denote by $F$ the closed subset $X \backslash U$ and by $\mathcal{I}_{F}$ the ideal of functions vanishing on $F$. Fix a positive integer $\gamma \geq 1$. For $n \geq 1$, we consider the constructible set

$$
\mathcal{X}_{n}^{\gamma n}(f, U):=\left\{\varphi \in \mathcal{L}_{\gamma n}(X) \mid \operatorname{ord}_{t} f(\varphi)=n, \operatorname{ord}_{t} \varphi^{*}\left(\mathcal{I}_{F}\right) \leq \gamma n\right\},
$$

we set $\mathcal{X}_{n}^{\gamma n, 1}(f, U)=\mathcal{X}_{n}^{\gamma n}(f, U) \cap f_{n}^{-1}(1)$ and define the modified zeta function as

$$
Z_{f, U}^{\gamma}(T):=\sum_{n \geq 1}\left[\mathcal{X}_{n}^{\gamma n, 1}(f, U)\right] \mathbb{L}^{-\gamma n d} T^{n}
$$

in $\mathcal{M}_{X_{0}(f)}^{\widehat{\mu}}[[T]]$. Note that for $U=X, Z_{f, U}^{\gamma}(T)$ is equal to $Z_{f}(T)$ for every $\gamma$, and that if $f$ vanishes on $X$ it is 0 . If $X_{0}(f)$ is nowhere dense in $X$, considering a log-resolution of $\left(X, F \cup X_{0}(f)\right)$, one proves in 14 that there exists $\gamma_{0}$ such that for every $\gamma>\gamma_{0}$ the series $Z_{f, U}^{\gamma}(T)$ is rational and admits a $\operatorname{limit}_{\lim } \lim _{T \rightarrow \infty} Z_{f, U}^{\gamma}(T)$ which is independent of $\gamma>\gamma_{0}$. Thus one may set

$$
\mathcal{S}_{f, U}:=-\lim _{T \rightarrow \infty} Z_{\gamma, U}^{\gamma}(T)
$$

for $\gamma \gg 0$. Note that $\mathcal{S}_{f, U}$ is an element of $\mathcal{M}_{X_{0}(f)}^{\widehat{\mu}}$.

We can then state

1.6. Theorem (Guibert-Loeser-Merle [14]). Let $X$ be a variety with a function $f: X \rightarrow \mathbb{A}_{k}^{1}$. There exists a unique $\mathcal{M}_{k}$-linear group morphism

$$
\mathcal{S}_{f}: \mathcal{M}_{X} \rightarrow \mathcal{M}_{X_{0}(f)}^{\widehat{\mu}}
$$


such that, for every proper morphism $p: Z \rightarrow X$ with $Z$ smooth, and every dense open subset $U$ in $Z$,

$$
\mathcal{S}_{f}([U \rightarrow X])=p_{!}\left(\mathcal{S}_{f \circ p, U}\right) .
$$

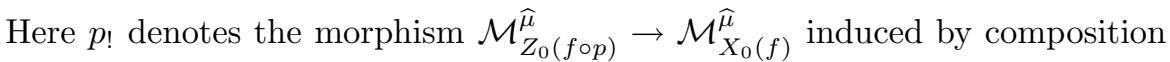
with $p$, with $Z_{0}(f \circ p)$ the zero locus of $f \circ p$ in $Z$. The construction in the previous theorem can be carried out equivariantly (cf. [14]), leading to a morphism

$$
\mathcal{S}_{f}: \mathcal{M}_{X}^{\widehat{\mu}} \rightarrow \mathcal{M}_{X_{0}(f)}^{\widehat{\mu} \times \widehat{\mu}} .
$$

The following result can be viewed as a motivic analogue of a conjecture by Steenbrink:

1.7. Theorem (Guibert-Loeser-Merle [14]). Let $X$ be a smooth variety and $f$ and $g$ be two functions from $X$ to $\mathbb{A}^{1}$. Let $x$ be a closed point of $X_{0}(f) \cap X_{0}(g)$. For $N \gg 0$, we have the equality

$$
\mathcal{S}_{f, x}^{\phi}-\mathcal{S}_{f+g^{N}, x}^{\phi}=\Psi_{\Sigma}\left(i_{x}^{*}\left(\mathcal{S}_{g^{N}}\left(\mathcal{S}_{f}^{\phi}\right)\right)\right)
$$

Here $\mathcal{S}_{g^{N}}\left(\mathcal{S}_{f}^{\phi}\right)$ lives in $\mathcal{M}_{X_{0}(f) \cap X_{0}(g)}^{\widehat{\mu} \times \widehat{ }}$ and $i_{x}^{*}$ stands for taking the fiber over $x$.

\section{$\S 1.7$. Some further open problems about the motivic Milnor fiber}

In their paper 22] on motivic Donaldson-Thomas invariants, Kontsevich and Soibelman propose the following conjecture which plays an important role in their program:

1.8. Conjecture (Kontsevich-Soibelman [22]). Let $W=V_{1} \times V_{2} \times V_{3}$ be the product of three finite-dimensional vector spaces over $k$. Let $F$ be a polynomial with $F(0,0,0)=0$. Assume that $F\left(\lambda v_{1}, \lambda^{-1} v_{2}, v_{3}\right)=F\left(v_{1}, v_{2}, v_{3}\right)$ for every $\lambda$ in $k^{\times}$. Set $f=F_{\mid V_{1} \times\{0\} \times\{0\}}$ and $g=F_{\mid\{0\} \times\{0\} \times V_{3}}$. Then

$$
\pi_{!} \mathcal{S}_{f}=\mathbb{L}^{\operatorname{dim} V_{1}} \mathcal{S}_{g,(0,0,0)},
$$

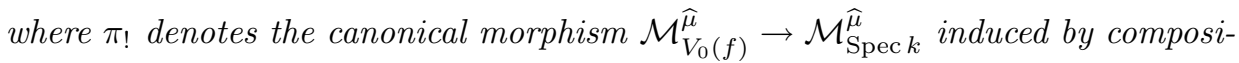
tion with the projection to the point, with $V_{0}(f)$ the zero locus of $f$ in $V_{1}$.

Note that in 22] the conjecture is stated under more general assumptions, in particular $F$ is assumed to be a formal series on $W$ depending in a constructible way on finitely many extra parameters.

Using results of Guibert, Merle and Loeser [15] and [16] on motivic Milnor fibers of compositions (cf. 1.5), Lê Quy Thuong [25] was recently able to prove some significant cases of Conjecture 1.8 . 
Note that motivic Milnor fibers also play a role in the recent work by Behrend, Bryan, and Szendrői on motivic degree-zero Donaldson-Thomas invariants [3.

\section{$\S 2$. Tangent cones and wave fronts over the $p$-adics}

\section{$\S 2.1$. Tangent cones over the $p$-adics}

In motivic integration, semi-algebraic (also known as definable) sets over $k((t))$ played a fundamental role already in [11] and then more recently again in [7]. Thus it is quite natural to try to develop microlocal geometry in that category, similarly to [21]. Before building such a theory from scratch, it seems useful to start by exploring the $p$-adic case as a toy model. We now present some of the $p$-adic results recently obtained in [5] and [6]. Extension of these results to $k((t))$ and the motivic world is part of some work in progress with Cluckers and Comte.

Over the $p$-adics, one can define semi-algebraic sets by mimicking the definition over the reals as follows. The Boolean algebra of semi-algebraic subsets of $\mathbb{Q}_{p}^{n}$ is the smallest such algebra containing sets of zeroes of polynomial functions and such that images of semi-algebraic subsets under linear projections $\mathbb{Q}_{p}^{n+1} \rightarrow \mathbb{Q}_{p}^{n}$ are still semi-algebraic. By a fundamental result of Macintyre [29], semi-algebraic $p$-adic sets may be described as Boolean combinations of zeroes of polynomial functions and of sets of the form " $f(x)$ is an $m$-th power" with $f$ a polynomial function and $m$ an integer. Remark that if one restricts to $m=2$, this statement is similar to Tarski's classical result over the reals.

Now, if one is willing to define the tangent cone at a point to a semi-algebraic subset one is faced with the issue of finding an adequate substitute for the multiplicative group of positive reals. The idea, which can be traced back to Heifetz [18, is to consider all finite index subgroups $\Lambda$ of $\mathbb{Q}_{p}^{\times}$at once. Thus, if $X$ is a semi-algebraic subset of $\mathbb{Q}_{p}^{n}, x$ a point of $\mathbb{Q}_{p}^{n}$, and $\Lambda$ a finite index subgroup of $\mathbb{Q}_{p}^{\times}$, one defines the tangent $\Lambda$-cone to $X$ at $x$ as

$$
\begin{aligned}
C_{x}^{\Lambda}(X):=\left\{u \in \mathbb{Q}_{p}^{n} \mid(\forall i>0)(\exists z\right. & \in X)(\exists \lambda \in \Lambda) \text { such that } \\
& \quad \operatorname{ord}(z-x)>i \text { and } \operatorname{ord}(\lambda(z-x)-u)>i\} .
\end{aligned}
$$

Here ord stands for the $p$-adic valuation. By construction $C_{x}^{\Lambda}(X)$ is a closed semialgebraic susbet. It is a $\Lambda$-cone, that is, it is stable under the canonical $\Lambda$-action.

This construction provides a whole bunch of tangent cones, so it is natural to ask whether there is one which is better than the others. The answer is that they stabilize when $\Lambda$ is small enough. This result, which we found unexpectedly difficult to prove, is one of the main results of $[6]$ : 
2.1. Theorem (Cluckers-Comte-Loeser [6]). Let $X$ be a semi-algebraic subset of $\mathbb{Q}_{p}^{n}$. Then there exists $\Lambda_{0}$ such that for every $x \in \bar{X}$, for every finite index subgroup $\Lambda$ of $\mathbb{Q}_{p}^{\times}$, we have $C_{x}^{\Lambda}(X)=C_{x}^{\Lambda_{0}}(X)$ whenever $\Lambda \subset \Lambda_{0}$.

The proof is quite involved and required the following result that can be considered as a $p$-adic substitute for the mean value theorem.

2.2. Theorem (Cluckers, Comte, Loeser [5]). Let $X$ be a semi-algebraic subset of $\mathbb{Q}_{p}^{n}$ and $f: X \rightarrow \mathbb{Q}_{p}$ be a semi-algebraic function (that is, one whose graph is semi-algebraic). Assume there is a constant $C>0$ such that $f$ is locally Lipschitz continuous with constant $C$ at each point of $X$. Then there is a finite partition of $X$ into semi-algebraic subsets $X_{i}$ and a constant $C^{\prime}>0$ such that the restriction of $f$ to each $X_{i}$ is globally Lipschitz continuous with constant $C^{\prime}$.

This is a $p$-adic analogue of a result by Kurdyka [23] over the reals. However, the proof by Kurdyka relies heavily on the use of paths connecting points and the relation between lenghts of paths and sizes of derivatives. Clearly such an approach cannot be followed over the $p$-adics, and the heart of the proof in [5] was to find appropriate $p$-adic substitutes.

Let us sketch the argument in [5] in the one-variable and two-variable cases. Let $g: X \subset \mathbb{Q}_{p} \rightarrow \mathbb{Q}_{p}$ be a semi-algebraic function which is locally Lipschitz continuous with constant $C$. We know that we can partition $X$ into finitely many $p$-adic cells, all of which are very roughly of a form similar to

$$
A=\left\{t \in \mathbb{Q}_{p}|| \alpha|\leq| t-c|\leq| \beta \mid, t-c \in \lambda Q_{m, n}\right\},
$$

with $\alpha, \beta, c$ and $\lambda$ in $\mathbb{Q}_{p}, n>0, m>0$, and where $Q_{m, n}$ is the set of all $p$-adic numbers of the form $p^{n a}\left(1+p^{m} x\right)$ for some $x \in \mathbb{Z}_{p}$ and some $a \in \mathbb{Z}$. One calls $c$ the center of $A$, even if $c$ may lie outside $A$, which happens precisely when $\lambda \neq 0$, and may not be uniquely determined by $A$. We define the balls of the cell $A$ as the collection of maximal balls (with respect to inclusion) contained in $A$. It then follows from a certain Jacobian property that we can select the cells $A$ in such a way that each ball of $A$ is mapped by $g$ to either a point or a ball. We refine this Jacobian property so that we can make sure that the images of the balls of $A$ form a single cell which has moreover as collection of maximal balls precisely the collection of the images of the maximal balls in $A$. In particular, $g(A)$ is a cell with a certain center $d$. Then, roughly, distances between points in $A$ are compared with distances to $c$ and similarly in the range of $g$, using the center $d$. In the one-variable case a computation based on this comparison of distances and the Jacobian property then allows one to conclude. 
In the two-variable case, one proceeds as follows. Let $g: X \subset \mathbb{Q}_{p}^{2} \rightarrow \mathbb{Q}_{p}$ be locally Lipschitz continuous with some constant $C$. Roughly, we partition the family $X_{x_{1}}:=\left\{x_{2} \mid\left(x_{1}, x_{2}\right) \in X\right\}$ into finitely many families of cells $A_{x_{1}} \subset \mathbb{Q}_{p}$ with center $c$ and boundaries $\alpha$ and $\beta$ now depending on $x_{1}$. We show that, after possibly switching the roles of $x_{1}$ and $x_{2}$, we can make sure that the center $c$ is Lipschitz continuous in $x_{1}$. By a piecewise bi-Lipschitz transformation, we may then assume that the center is identically zero for each of the cells. This is already a significant step, but the issues due to the lack of a good notion of paths and integrals to control distances remain. Instead of working with paths as in the real case, we work with a finite sequence of points with given starting point and endpoint. One should view such a finite sequence of jumps from one point to the closest one as a $p$-adic analogue of a real "path". For such a sequence of jumps to be of use, the following is required: after each jump, one should remain in the same cell so that one can still evaluate the function $g$, the total cumulative distance of the jumps should be comparable to the distance between the starting point and the endpoint, and the function should not vary too much at each jump so that one can control $|g(a)-g(b)|$ for any jump from $a$ to $b$ in the sequence. This is achieved as follows. Let $a$ and $a^{\prime}$ be given in $A$. Either $\alpha\left(x_{1}\right)$, where we view $\alpha$ as a function of the parameter $x_{1}$, has bounded derivative, and then we can use induction for the one-variable function $x_{1} \mapsto g\left(x_{1}, \alpha\left(x_{1}\right)\right)$ and roughly jump from $a$ to $\left.\left(a_{1}, \alpha\left(a_{1}\right)\right)\right)$, then to $\left.\left(a_{1}^{\prime}, \alpha\left(a_{1}^{\prime}\right)\right)\right)$ and finally to $a^{\prime}$. In the seemingly more difficult case where $\alpha\left(x_{1}\right)$ has large derivative, we switch the roles of $x_{1}$ and $x_{2}$ in the parametrization of the function $g\left(x_{1}, \alpha\left(x_{1}\right)\right)$, namely, we essentially deal with the one-variable function $x_{2} \mapsto g\left(\alpha^{-1}\left(x_{2}\right), x_{2}\right)$ and use induction for this function and then roughly make similar jumps as before: from $a$ to $\left.\left(a_{1}, \alpha\left(a_{1}\right)\right)\right)=\left(\alpha^{-1}(b), b\right)$, then to $\left.\left(\alpha^{-1}\left(b^{\prime}\right), b^{\prime}\right)=\left(a_{1}^{\prime}, \alpha\left(a_{1}^{\prime}\right)\right)\right)$ and finally to $a^{\prime}$, for some $b, b^{\prime} \in \mathbb{Q}_{p}$. Such a "path" allows us to bound $\left|g(a)-g\left(a^{\prime}\right)\right|$ in terms of $\left|a-a^{\prime}\right|$ as needed for Lipschitz continuity, uniformly in $a$ and $a^{\prime}$ in the cell.

\section{$\S 2.2$. Wave fronts}

Let $V$ be a finite-dimensional vector space over $\mathbb{Q}_{p}$. If $U$ is an open subset of $V$, the space $\mathcal{S}(U)$ of $S$ chwartz-Bruhat functions on $V$ is the space of locally constant complex valued functions with compact support in $U$. Given an additive character $\Psi$ on $\mathbb{Q}_{p}$ with conductor $\mathbb{Z}_{p}$, the Fourier transform $\mathcal{F}(f)$ of a function $f$ in $\mathcal{S}(V)$ is defined by

$$
\mathcal{F}(f)(y)=\int_{V} f(x) \Psi(\langle x, y\rangle)|d x|,
$$


with $|d x|$ a Haar measure. It is a Schwartz-Bruhat function on the dual space $V^{*}$ and $\langle\cdot, \cdot\rangle$ denotes the duality pairing. One defines the space $\mathcal{S}^{\prime}(U)$ of tempered distributions on $U$ as the linear dual of $\mathcal{S}(U)$.

In 18, Heifetz defines a notion of wave front for tempered $p$-adic distributions as follows:

2.3. Definition. Let $U$ be an open subset of $V$ and $u$ in $\mathcal{S}^{\prime}(U)$. Let $\Lambda$ be a finite index subgroup of $\mathbb{Q}_{p}^{\times}$. One says $u$ is $\Lambda$-smooth at $\left(x_{0}, \xi_{0}\right) \in U \times V \backslash\{0\}$ if there exist open neighborhoods $O$ of $x_{0}$ and $O^{\prime}$ of $\xi_{0}$ such that, for every $\varphi$ in $\mathcal{S}(O)$, there exists $N>0$ such that, for every $\lambda>N$ in $\Lambda, \mathcal{F}(\varphi u)(\lambda \xi)=0$ for every $\xi$ in $O^{\prime}$.

2.4. Definition. Let $U$ be an open subset of $V$ and $u$ in $\mathcal{S}^{\prime}(U)$. Let $\Lambda$ be a finite index subgroup of $\mathbb{Q}_{p}^{\times}$. One defines the wave front $W F_{\Lambda}(u)$ as the complement of the set of smooth directions.

Now if $X$ is an $n$-dimensional $\mathbb{Q}_{p}$-analytic manifold, the definitions of $\mathcal{S}(X)$ and $\mathcal{S}^{\prime}(X)$ globalize. If $u$ belongs to $\mathcal{S}^{\prime}(X)$ one checks that the definition of $W F_{\Lambda}$ can be globalized to give a closed $\Lambda$-cone $W F_{\Lambda}(u)$ in $T^{*}(X) \backslash\{0\}$.

In his paper, Heifetz proves that $W F_{\Lambda}$ satisfies the usual functorial properties with respect to pull-backs, push-forwards, etc.

As for motivic counterparts, we have now at hand a motivic formalism allowing us to construct $W F_{\Lambda}$ at the motivic level. More precisely, one replaces $\mathbb{Q}_{p}$ by $k((t))$, with $k$ a field of characteristic zero. In [8], motivic versions of $\mathcal{S}(V)$ and $\mathcal{F}$ were constructed, relying on the constructions of [7] (see also [19]). The space $\mathcal{S}^{\prime}$ is still defined as the linear dual of $\mathcal{S}$. Hence, it is already possible to develop full motivic analogues of the results of [18, which we intend to do in the near future.

\section{Acknowledgements}

This work was partially supported by ANR grant 06-BLAN-0183.

\section{References}

[1] A. Abbes and T. Saito, Analyse micro-locale $\ell$-adique en caractéristique $p>0$ : le cas d'un trait, Publ. Res. Inst. Math. Sci. 45 (2009), 25-74. Zbl pre05551347 MR 2512777

[2] D. Barlet and H. Maire, Asymptotique des intégrales-fibres, Ann. Inst. Fourier (Grenoble) 43 (1993), 1267-1299. Zbl 0803.32004 MR 1275199

[3] K. Behrend, J. Bryan and B. Szendrői, Motivic degree zero Donaldson-Thomas invariants, arXiv:0909.5088.

[4] F. Bittner, On motivic zeta functions and the motivic nearby fiber, Math. Z. 249 (2005), 63-83. Zbl 1085.140201085.14020 
[5] R. Cluckers, G. Comte and F. Loeser, Lipschitz continuity properties for p-adic semialgebraic and subanalytic functions, Geom. Funct. Anal. 20 (2010), 68-87. Zbl pre05770215 MR 2647135

[6] Local metric properties and regular stratifications of $p$-adic definable sets, Comment. Math. Helv., to appear; arXiv:0910.0799.

[7] R. Cluckers and F. Loeser, Constructible motivic functions and motivic integration, Invent. Math. 173 (2008), 23-121. Zbl 1179.14011 MR 2403394

[8] Constructible exponential functions, motivic Fourier transform and transfer principle, Ann. of Math. 171 (2010), 1011-1065. Zbl pre05712748 MR 2630060

[9] J. Denef, Degree of local zeta functions and monodromy, Compos. Math. 89 (1993), 207-216. Zbl 0932.11073 MR 1255694

[10] J. Denef and F. Loeser, Motivic Igusa zeta functions, J. Algebraic Geom. 7 (1998), 505-537. Zbl 0943.14010 MR 1618144

[11] Germs of arcs on singular algebraic varieties and motivic integration, Invent. Math. 135 (1999), 201-232. Zbl 0928.14004 MR 1664700

[12] Motivic exponential integrals and a motivic Thom-Sebastiani theorem, Duke Math. J. 99 (1999), 285-309. Zbl 0966.14015 MR 1708026

[13] Geometry on arc spaces of algebraic varieties, in Proc. 3rd European Congress of Mathematics (Barcelona, 2000), Progr. Math. 201, Birkhäuser, 2001, 327-348. Zbl 1079.14003 MR 1905328

[14] G. Guibert, F. Loeser and M. Merle, Iterated vanishing cycles, convolution, and a motivic analogue of a conjecture of Steenbrink, Duke Math. J. 132 (2006), 409-457. Zbl 1173.14301 MR 2219263

[15] N Nearby cycles and composition with a non-degenerate polynomial, Int. Math. Res. Notices 2005, no. 31, 1873-1888. Zbl 1093.14032 MR 2171196

[16] Composition with a two variable function, Math. Res. Lett. 16 (2009), 439-448. Zbl 1187.14046 MR 2511624

[17] L. Halle and J. Nicaise, Motivic zeta functions of abelian varieties, and the monodromy conjecture, Adv. Math. 227 (2011), 610-653. Zbl pre05880836

[18] D. Heifetz, p-adic oscillatory integrals and wave front sets, Pacific J. Math. 116 (1985), 285-305. Zbl 0528.22008 MR 0771637

[19] E. Hrushovski and D. Kazhdan, Integration in valued fields, in Algebraic geometry and number theory, Progr. Math. 253, Birkhäuser, 2006, 261-405. Zbl 1136.03025 MR 2263194

[20] E. Hrushovski and F. Loeser, Non-archimedean tame topology and stably dominated types, arXiv:1009.0252.

[21] M. Kashiwara and P. Schapira, Sheaves on manifolds, Grundlehren Math. Wiss. 292, Springer, Berlin, 1990. Zbl 0709.18001 MR 1074006

[22] M. Kontsevich and Y. Soibelman, Stability structures, motivic Donaldson-Thomas invariants and cluster transformations, arXiv:0811.2435.

[23] K. Kurdyka, On a subanalytic stratification satisfying a Whitney property with exponent 1, in Real algebraic geometry (Rennes, 1991), Lecture Notes in Math. 1524, Springer, Berlin, 1992, 316-322. Zbl 0779.32006 MR 1226263

[24] Lê Dung Tráng, The geometry of the monodromy theorem, in C. P. Ramanujam-a tribute, Tata Inst. Fund. Res. Stud. Math. 8, Springer, Berlin, 1978, 157-173. Zbl 0434.32010 MR 0541020

[25] Lê Quy Thuong, On a conjecture of Kontsevich and Soibelman, arXiv:1004.1311.

[26] A. Lemahieu and L. Van Proeyen, Monodromy conjecture for nondegenerate surface singularities, preprint. 
[27] F. Loeser, Fonctions zêta locales d'Igusa à plusieurs variables, intégration dans les fibres, et discriminants, Ann. Sci. École Norm. Sup. 22 (1989), 435-471. Zbl 0718.11061 MR 1011989

[28] E. Looijenga, Motivic measures, in Séminaire Bourbaki, Vol. 1999/2000, Astérisque 276 (2002), 267-297. Zbl 0996.14011 MR 1886763

[29] A. Macintyre, On definable subsets of $p$-adic fields, J. Symbolic Logic 41 (1976), 605-610. Zbl 0362.02046 MR 0485335

[30] A. Némethi, The zeta function of singularities, J. Algebraic Geom. 2 (1993), 1-23. Zbl 0778.32011 MR 1185605

[31] A. Némethi and J. Steenbrink, Spectral pairs, mixed Hodge modules, and series of plane curve singularities, New York J. Math. 1 (1994/95), 149-177. Zbl 0878.14017| MR 1343394

[32] C. Sabbah, Proximité évanescente. II. Équations fonctionnelles pour plusieurs fonctions analytiques, Compos. Math. 64 (1987), 213-241. Z Zbl $0632.32006 \mid$ MR 0916482

[33] T. Saito, Wild ramification and the characteristic cycle of an $\ell$-adic sheaf, J. Inst. Math. Jussieu 8 (2009), 769-829. Zbl 1177.14044 MR 2540880 